\title{
On Graphs Having no Flow Roots in the Interval $(1,2)$
}

\author{
F.M. Dong* \\ Mathematics and Mathematics Education \\ National Institute of Education \\ Nanyang Technological University, Singapore 637616 \\ fengming.dong@nie.edu.sg
}

Submitted: Oct 24, 2013; Accepted: Mar 17, 2015; Published: Mar 30, 2015

Mathematics Subject Classifications: 05C25, O5C31

\begin{abstract}
For any graph $G$, let $W(G)$ be the set of vertices in $G$ of degrees larger than 3 . We show that for any bridgeless graph $G$, if $W(G)$ is dominated by some component of $G-W(G)$, then $F(G, \lambda)$ has no roots in $(1,2)$, where $F(G, \lambda)$ is the flow polynomial of $G$. This result generalizes the known result that $F(G, \lambda)$ has no roots in $(1,2)$ whenever $|W(G)| \leqslant 2$. We also give some constructions to generate graphs whose flow polynomials have no roots in $(1,2)$.
\end{abstract}

Keywords: chromatic polynomial, flow polynomial

\section{Introduction}

The graphs considered in this paper are undirected and finite, and may have loops and parallel edges. However, the graphs should have no loops when their chromatic polynomials are considered, and the graphs should have no bridges when their flow polynomials are considered. For any graph $G$, let $V(G), E(G), P(G, \lambda)$ and $F(G, \lambda)$ be the set of vertices, the set of edges, the chromatic polynomial and the flow polynomial of $G$. The roots of $P(G, \lambda)$ and $F(G, \lambda)$ are called the chromatic roots and the flow roots of $G$ respectively.

A near-triangulation is a loopless connected plane graph in which at most one face is not bounded by a cycle of order 3. Birkhoff and Lewis [1] showed that $G$ has no real chromatic roots in $(1,2)$ for every near-triangulation $G$. Since $P(G, \lambda)=\lambda F\left(G^{*}, \lambda\right)$ for any plane graph $G$, where $G^{*}$ is its dual, this result is equivalent to that any connected plane graph $G$ has no flow roots in $(1,2)$ under the condition $|W(G)| \leqslant 1$, where $W(G)$ is

${ }^{*}$ Partially supported by NIE AcRf funding (RI 2/12 DFM) of Singapore and the National Natural Science Foundation of China (No.11271307). 
the set of vertices $x$ in $G$ with its degree larger than 3 and the degree of $x$ in $G$, denoted by $d_{G}(x)$ (or simply $d(x)$ ), is defined to be the sum of the number of non-loop edges in $G$ incident with $x$ and twice the number of loops in $G$ incident with $x$.

Jackson [5] generalized Birkhoff and Lewis' result by showing that any bridgeless connected graph $G$ with $|W(G)| \leqslant 1$ has no real flow roots in $(1,2)$, no matter whether $G$ is planar or non-planar. This result was further generalized by Dong [2]. For any integer $k \geqslant 0$, let $\Psi_{k}$ be the set of bridgeless connected graphs with $|W(G)| \leqslant k$ and $\xi_{k}$ be the supremum in $(1,2]$ such that every graph $G$ in $\Psi_{k}$ has no flow roots in $\left(1, \xi_{k}\right)$. It was shown in [2] that $\xi_{2}=2$. But it is also shown there that $\xi_{k}<2$ for all $k \geqslant 3$, i.e., $\Psi_{k}$ contains bridgeless connected graphs with flow roots in $(1,2)$ for all $k \geqslant 3$. For example, the graph in Figure 1 belongs to $\Psi_{3}$ and has a real flow root $1.430159709 \ldots$ which is the only zero of $\lambda^{3}-5 \lambda^{2}+10 \lambda-7$ in $(1,2)$.

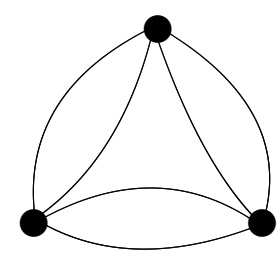

Figure 1: A graph in $\Phi_{3}$ having flow zeros in $(1,2)$

The main purpose of this paper is to find graphs in each set $\Psi_{k}$ which have no flow roots in $(1,2)$, although we are not able to determine all such graphs. For any vertex $x$ in $G=(E, V)$, let $N_{G}(x)$ (or simply $N(x)$ ) denote the set of vertices in $G$ which are adjacent to $x$. Thus $d(x) \geqslant|N(x)|$, where the equality holds if and only if no loops and no parallel edges are incident with $x$. For any graph $G$ and $S \subseteq V(G)$, let $N_{G}(S)$ (or simply $N(S)$ ) be the set defined below:

$$
N_{G}(S)=\bigcup_{x \in S}(N(x) \backslash S)
$$

For any subgraph $H$ of $G$, let $N_{G}(H)=N_{G}(V(H))$. Any subset of $N_{G}(H)$ is said to be dominated by $H$. Recall that $W(G)=\{x \in V(G): d(x) \geqslant 4\}$. Let $\Upsilon$ denote the family of graphs $G$ satisfying the condition that either $|V(G)| \leqslant 2$ or $W(G)$ is dominated by some component of $G-W(G)$, where $G-W(G)$ is the subgraph of $G$ induced by $V(G)-W(G)$. Note that $W(G)$ is dominated by some component of $G-W(G)$ if and only if $W(G)$ is dominated by a connected subgraph of $G-W(G)$.

In Section 2, we introduce some known results which will be applied in Sections 3 and 4. In Section 3, we show that all bridgeless graphs in $\Upsilon$ have no flow roots in $(1,2)$. In Section 4, we provide two constructions to generate graphs which have no flow roots in $(1,2)$. Some graphs produced by these constructions do not belong to $\Upsilon$.

Note that this article does not study real flow roots larger than 4. Recently, Jacobsen and Salas [7] proved that there is a sequence of real flow roots that converges to 5 from below and also showed that there exist real flow roots larger than 5 . 


\section{Preliminary results}

The flow polynomial $F(G, \lambda)$ of a graph $G$ can be obtained from the following properties of $F(G, \lambda)$ (see Tutte $[11])$ :

$$
F(G, \lambda)= \begin{cases}1, & \text { if } E=\emptyset \\ F\left(G_{1}, \lambda\right) F\left(G_{2}, \lambda\right), & \text { if } G=G_{1} \cup G_{2} \\ 0, & \text { if } G \text { has a bridge; } \\ (\lambda-1) F(G-e, \lambda), & \text { if } e \text { is a loop; } \\ F(G / e, \lambda)-F(G-e, \lambda), & \text { if } e \text { is not a loop nor a bridge }\end{cases}
$$

where $e$ is an edge of $G, G-e$ and $G / e^{1}$ are the graphs obtained from $G$ by deleting $e$ and contracting $e$ respectively, and $G_{1} \cup G_{2}$ is the disjoint union of graphs $G_{1}$ and $G_{2}$.

A graph $G=(V, E)$ is said to be non-separable if it is connected, has no cut-vertex and either $G$ has no loops or $|E|=|V|=1$, where a vertex $x$ in $G$ is called a cut-vertex if $G-x$, the graph obtained from $G$ by deleting $x$ and all edges incident with $x$, has more components that $G$ has. A graph is said to be separable if it is not non-separable. So every loopless connected graph $G$ with $|V(G)| \leqslant 2$ is non-separable, and a non-separable graph has a bridge if and only if this graph is $K_{2}$.

For any graph $G$, a block of $G$ is a maximal subgraph of $G$ with the property that it is non-separable. So every loop is also considered as a block, and any block with more than one vertex has no loops nor cut-vertices. Let $b(G)$ be the number of blocks $B$ of $G$ with $E(B) \neq \emptyset$. When $b(G)=1, G$ does not need to be non-separable as it is possible that $G$ is not connected. If $G$ is connected, then $b(G)=1$ if and only if $G$ is non-separable with $E(G) \neq \emptyset$.

For a connected graph $G=(V, E)$ without loops, it is well known (see Woodall [10]) that $(-1)^{|V|} P(G, \lambda)>0$ for all real $\lambda<0$ and $(-1)^{|V|-1} P(G, \lambda)>0$ for all real $0<\lambda<1$. Woodall [10] and Whitehead and Zhao [9] independently showed that $G$ always has a chromatic root of multiplicity $b(G)$ at $\lambda=1$. Jackson [3] also proved that $(-1)^{|V|-b(G)+1} P(G, \lambda)>0$ for all real $1<\lambda \leqslant 32 / 27$, where $32 / 27$ cannot be replaced by any larger number. There is an analogous result for flow polynomials due to Wakelin [8].

Theorem 1 ([8]). Let $G=(V, E)$ be a bridgeless connected graph. Then

(a) $F(G, \lambda)$ is non-zero with sign $(-1)^{|E|-|V|+1}$ for $\lambda \in(-\infty, 1)$;

(b) $F(G, \lambda)$ has a zero of multiplicity $b(G)$ at $\lambda=1$;

(c) $F(G, \lambda)$ is non-zero with sign $(-1)^{|E|-|V|+b(G)-1}$ for $\lambda \in(1,32 / 27]$.

By (2), the following result can be easily proved by induction.

\footnotetext{
${ }^{1}$ If $u$ and $v$ are two vertices of a graph $H$, let $H / u v$ denote the graph obtained from $H$ by identifying $u$ and $v$. So every edge of $H$ is also an edge in $H / u v$ and every edge of $H$ joining $u$ and $v$ becomes a loop in $H / u v$. Then $G / e$ is the graph $(G-e) / u v$, where $u$ and $v$ are the two ends of $e$.
} 
Lemma 2. Let $G$ be a bridgeless graph. If $G_{1}, G_{2}, \ldots, G_{k}$ are the components of $G$ or $G_{1}, G_{2}, \ldots, G_{k}$ are the blocks of $G$, then

$$
F(G, \lambda)=\prod_{1 \leqslant i \leqslant k} F\left(G_{i}, \lambda\right)
$$

The next result on the factorization of flow polynomials can be found in [5] (see $[4,6]$ also). For any graph $G$ and any two vertices $u$ and $v$ in $G$, let $G+u v$ denote the graph obtained by adding a new edge joining $u$ and $v$.

Lemma $3([5])$. Let $G$ be a bridgeless connected graph, $v$ be a vertex of $G, e=u_{1} u_{2}$ be an edge of $G$, and $H_{1}$ and $H_{2}$ be edge-disjoint subgraphs of $G$ such that $E\left(H_{1}\right) \cup E\left(H_{2}\right)=$ $E(G-e), V\left(H_{1}\right) \cap V\left(H_{2}\right)=\{v\}, V\left(H_{1}\right) \cup V\left(H_{2}\right)=V(G), u_{1} \in V\left(H_{1}\right)$ and $u_{2} \in V\left(H_{2}\right)$, as shown in Figure 2. Then

$$
F(G, \lambda)=\frac{F\left(G_{1}, \lambda\right) F\left(G_{2}, \lambda\right)}{\lambda-1} .
$$

where $G_{i}=H_{i}+$ vu $u_{i}$ for $i \in\{1,2\}$.

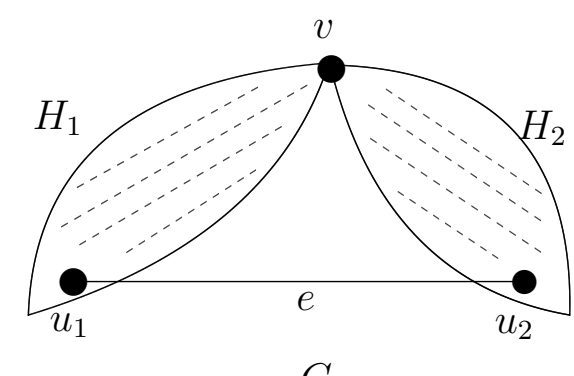

$G$

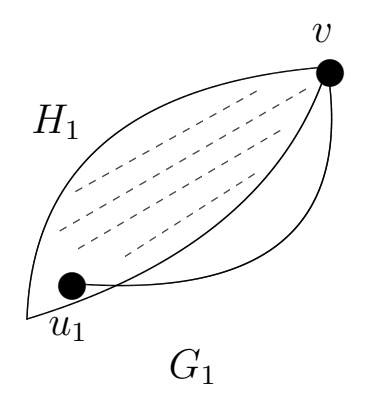

$G_{1}$

Figure 2: $\quad G-e$ is separable.

The following result given in [2] can be easily proved by applying the recursive expression in (2) and Lemma 3. It will be applied latter.

Lemma 4 ([2]). Let $G$ be a non-separable graph with edge-disjoint subgraphs $G_{1}$ and $G_{2}$ such that $V\left(G_{1}\right) \cap V\left(G_{2}\right)=\{u, v\}, V\left(G_{1}\right) \cup V\left(G_{2}\right)=V(G)$ and $E\left(G_{1}\right) \cup E\left(G_{2}\right)=E(G)$, as shown in Figure $3(a)$. Then

$$
F(G, \lambda)=\frac{F\left(G_{1}+u v, \lambda\right) F\left(G_{2}+u v, \lambda\right)}{\lambda-1}+F\left(G_{1}, \lambda\right) F\left(G_{2}, \lambda\right),
$$

where $u$ and $v$ be two vertives of $G$.

For any connected graph $G$, let

$$
Q(G, \lambda)=(-1)^{p(G)} F(G, \lambda)
$$

where $p(G)=|E(G)|-|V(G)|+b(G)-1$. So $p(G)=|E(G)|-|V(G)|$ if $G$ is non-separable with $E(G) \neq \emptyset$. It is clear that $F(G, \lambda) \neq 0$ if and only if $Q(G, \lambda) \neq 0$. Theorem 1 implies that $Q(G, \lambda)>0$ for any bridgeless connected graph $G$ and real number $\lambda \in(1,32 / 27$. Thus we have the following result. 


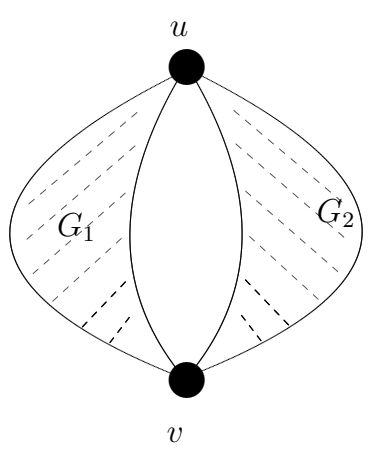

(a) $G$

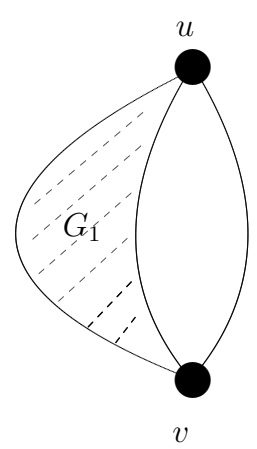

(b) $G_{1}+u v$

Figure 3: $\quad G$ is formed by $G_{1}$ and $G_{2}$

Corollary 5. Let $G$ be any bridgeless graph. Then $G$ has no flow roots in $(1,2)$ if and only if $Q(G, \lambda)>0$ for all $\lambda \in(1,2)$.

The following result is from Theorem 3.1 in [2]. It provides a sufficient condition for a family $\mathcal{S}$ of graphs to have no flow zeros in $(1, \beta)$ for some $\beta$ with $1<\beta \leqslant 2$. This result will be applied in the next section to prove the main result of this paper.

Theorem 6 (Theorem 3.1, [2]). Let $\mathcal{S}$ be a family of bridgeless connected graphs and $\beta$ a real number in $(1,2]$. Assume that there exists $\mathcal{S}^{\prime} \subseteq \mathcal{S}$ such that all the following three conditions are satisfied:

(i) $Q(G, \lambda)>0$ for all graphs $G \in \mathcal{S}^{\prime}$ and all real $\lambda \in(1, \beta)$;

(ii) for every separable graph $G \in \mathcal{S}$, all blocks of $G$ belong to $\mathcal{S}$;

(iii) for every non-separable graph $G \in \mathcal{S} \backslash \mathcal{S}^{\prime}$, one of the following cases occurs:

(a) for some edge e in $G, G-e$ has a cut-vertex $u$ and each $G_{i}$ belongs to $\mathcal{S}$ for $i=1,2$, where $G_{1}$ and $G_{2}$ are graphs stated in Lemma 3;

(b) for some edge e in $G$, both $G-e$ and $G / e$ belong to $\mathcal{S}$ and both $b(G-e)$ and $b(G / e)$ are odd numbers;

(c) there are subgraphs $G_{1}$ and $G_{2}$ of $G$ with $V\left(G_{1}\right) \cap V\left(G_{2}\right)=\{u, v\}, V\left(G_{1}\right) \cup$ $V\left(G_{2}\right)=V(G), E\left(G_{1}\right) \cap E\left(G_{2}\right)=\emptyset$ and $E\left(G_{1}\right) \cup E\left(G_{2}\right)=E(G)$, as shown in Figure 3(a), such that $b\left(G_{1}\right)+b\left(G_{2}\right)$ is even, and for $i=1,2,\left|E\left(G_{i}\right)\right| \geqslant 2$ and both $G_{i}+u v$ and $G_{i}$ belong to $\mathcal{S}$, where $G_{i}+u v$ is the graph obtained from $G_{i}$ by adding a new edge joining $u$ and $v$; and

Then $Q(G, \lambda)>0$ for all graphs $G \in \mathcal{S}$ and all real $\lambda \in(1, \beta)$.

Note that Theorem 3.1 in [2] has a weaker condition than Theorem 6 here, as Theorem 3.1 in [2] contains case (d) for Condition (iii). However, in the application of Theorem 3.1 of [2] in this paper, one of the three cases in Condition (iii) (i.e., (a), (b) and (c)) always occurs. Thus it is not necessary for Theorem 6 to include case (d) in Condition (iii). 


\section{A family of graphs having no flow roots in $(1,2)$}

Recall that $\Upsilon$ denotes the family of graphs $G$ satisfying the condition that either $|V(G)| \leqslant$ 2 or $W(G)$ is dominated by some component of $G-W(G)$. In this section, we will show that every bridgeless graph in $\Upsilon$ has no flow roots in $(1,2)$, which generalizes the result that all graphs in $\Psi_{2}$ has no flow roots in $(1,2)$, as the result below shows that $\Psi_{2} \subseteq \Upsilon$ and $\Psi_{k} \cap \Upsilon \neq \emptyset$ for every $k \geqslant 3$.

Lemma 7. $\Psi_{2} \subseteq \Upsilon$ and $\Psi_{k} \cap \Upsilon \neq \emptyset$ for every $k \geqslant 3$.

Proof. Let $G \in \Psi_{2}$. We need only to consider the case that $|V(G)| \geqslant 3$. As $G$ is connected, if $|W(G)| \leqslant 1$, then $W(G)$ is certainly dominated by every component of $G-W(G)$. If $|W(G)|=2$ and $W(G)$ is not dominated by any component of $W(G)$, then $G$ should be disconnected or the edge joining the two vertices of $W(G)$ is a bridge of $G$, contradicting the definition of $\Psi_{2}$.

Let $k \geqslant 3$. Consider the graph $G_{k}$ obtained from a $k$-cycle $C$ with vertices $v_{1}, v_{2}, \ldots, v_{k}$ and the complete graph $K_{k}$ with vertices $u_{1}, u_{2}, \ldots, u_{k}$ by adding an edge joining $v_{i}$ and $u_{i}$ for all $i=1,2, \ldots, k$. If $k \geqslant 4$, then $G_{k} \in \Psi_{k}$ and $W\left(G_{k}\right)=\left\{u_{i}: 1 \leqslant i \leqslant k\right\}$ is dominated by the cycle $C$. Thus $G_{k} \in \Upsilon$. If $k=3$, let $G_{3}^{\prime}$ be the graph obtained from $G_{3}$ by adding a new vertex $w$ and new edges joining $w$ to $u_{i}$ for all $i=1,2,3$. Then $G_{3}^{\prime} \in \Psi_{3} \cap \Upsilon$.

Some properties on graphs in $\Upsilon$ can be proved directly from the definition of $\Upsilon$. These properties will be applied later.

Lemma 8. Assume $H$ is a component of $G-W(G)$ such that $W(G) \subseteq N(H)$. Then the following results hold.

(i) For any edge subset $E_{0}$ and any vertex subset $V_{0}$ in the subgraph of $G$ induced by $W(G)$, both $G / E_{0}$ and $G-E_{0}-V_{0}$ belong to $\Upsilon$.

(ii) For any $x \in V \backslash W(G)$, if $x \notin V(H)$, then $G-x \in \Upsilon$; otherwise, if $x$ is not a cut-vertex of $H$ and $N(x) \cap W(G) \subseteq N_{G}(H-x)$, then $G-x \in \Upsilon$.

(iii) For any $e \in E$ with ends $x$ and $y$ not in $W(G)$, if $d(x) \leqslant 2$, then $G / e \in \Upsilon$; if $e$ is not a bridge of $H$, then $G-e \in \Upsilon$.

(iv) If $e_{1}, e_{2}$ are parallel edges, then $G-e_{1} \in \Upsilon$.

(v) Every component (or block) of $G$ belongs to $\Upsilon$.

Proof. Note that if $|V(G)| \leqslant 2$, all these properties hold. Thus we assume that $|V(G)| \geqslant$ 3.

Observe that $H$ is a connected subgraph of $G / E_{0}$ and $W\left(G / E_{0}\right)$ is dominated by $H$ in $G / E_{0}$. Thus $G / E_{0} \in \Upsilon$. Similarly, we also have $G-E_{0}-V_{0} \in \Upsilon$. Thus 1 holds by definition.

Let $x \in V \backslash W(G)$. If $x \notin V(H)$, then $W(G-x) \subseteq W(G)$ and $H$ is a subgraph of a component $H^{\prime}$ of $G-x-W(G-x)$. Thus $W(G-x) \subseteq N_{G-x}\left(H^{\prime}\right)$, implying that $G-x \in \Upsilon$. 
Now assume that $x \in V(H)$ is not a cut-vertex of $H$ and $N(x) \cap W(G) \subseteq N_{G}(H-x)$. Thus $H-x$ is connected and $W(G) \subseteq N_{G}(H-x)$. Observe that $W(G-x) \subseteq W(G)$ and $H-x$ is a subgraph of some component $H^{\prime}$ of $G-x$. Hence $W(G-x) \subseteq N_{G-x}\left(H^{\prime}\right)$, implying that $G-x \in \Upsilon$. So 2 holds.

For $e \in E$ with ends $x$ and $y$, if $x, y \notin W(G)$ and $d(x) \leqslant 2$, then the new vertex obtained after contracting $e$ has a degree less than 4 and all other vertices remain the same degrees. Thus $W(G / e)=W(G)$ and $W(G / e)$ is still dominated by a component $H^{\prime}$ of $G / e-W(G / e)$, where $H^{\prime}=H / e$ when $x, y \in V(H)$ and $H^{\prime}=H$ otherwise. If $e$ is not a bridge of $H$, then $H-e$ is a connected subgraph of $G-e$ and $W(G-e)=W(G) \subseteq$ $N_{G}(H)=N_{G-e}(H-e)$. So 3 holds.

If $e_{1}$ and $e_{2}$ are parallel edges in $G$, then 1 and 3 implies that $G-e_{1} \in \Upsilon$ if both ends of $e_{1}$ are in $W(G)$ or both ends are in $V \backslash W(G)$. If $e_{1}$ has one end in $W(G)$ only, then $H$ is a connected subgraph in $G-e_{1}$ and $W\left(G-e_{1}\right) \subseteq W(G) \subseteq N(H)$, implying that $G-e_{1} \in \Upsilon$. So 4 holds.

If $G_{0}$ is a component of $G$ and $V\left(G_{0}\right) \cap V(H)=\emptyset$, then $W\left(G_{0}\right)=\emptyset$ and so $G_{0} \in \Upsilon$ by definition. If $V\left(G_{0}\right) \cap V(H) \neq \emptyset$, then $H$ is a component of $G_{0}-W\left(G_{0}\right)=G_{0}-W(G)$ and $W\left(G_{0}\right)=W(G) \subseteq N(H)$, implying that $G_{0} \in \Upsilon$.

Now we assume that $G$ is connected and separable. Let $u$ be any cut-vertex of $G$ and $G_{1}$ and $G_{2}$ be edge-disjoint connected subgraphs of $G$ such that $V\left(G_{1}\right) \cup V\left(G_{2}\right)=V(G)$, $V\left(G_{1}\right) \cap V\left(G_{2}\right)=\{u\}$ and $E\left(G_{1}\right) \cup E\left(G_{2}\right)=E(G)$. It suffices to show that $G_{i} \in \Upsilon$ for both $i=1,2$. Let $i=1$ or $i=2$. It is obvious that $G_{i} \in \Upsilon$ if $W(G) \cap V\left(G_{i}-u\right)=\emptyset$. Now suppose that $W(G) \cap V\left(G_{i}-u\right) \neq \emptyset$. Since $W(G) \subseteq N(H)$ and $H$ is connected, $W(G) \cap V\left(G_{3-i}-u\right)=\emptyset$. Note that $G_{i}$ can be obtained from $G$ by removing all vertices in $V\left(G_{3-i}-u\right)$. Then applying result (ii) repeatedly yields that $G_{i} \in \Upsilon$.

Hence 5 holds.

Lemma 9. Let $G$ be any graph in $\Upsilon$ with edge-disjoint proper subgraphs $G_{1}$ and $G_{2}$ such that $V\left(G_{1}\right) \cap V\left(G_{2}\right)=\{u, v\}, V\left(G_{1}\right) \cup V\left(G_{2}\right)=V(G)$ and $E\left(G_{1}\right) \cup E\left(G_{2}\right)=E(G)$, as shown in Figure 3(a). For $i=1,2$, if there exist $u-v$ paths in $G_{3-i}$, then $G_{i}+u v \in \Upsilon$.

Proof. Suppose that there is a graph $G \in \Upsilon$ with subgraphs $G_{1}$ and $G_{2}$ stated in the lemma such that $G_{2}$ has a $u-v$ path but $G_{1}+u v \notin \Upsilon$. We further assume that $G$ is such a graph with the minimum number of edges. By definition, we have $\left|W\left(G_{1}+u v\right)\right| \geqslant 3$. So there is at least one vertex, say $w$, contained in $W\left(G_{1}+u v\right) \backslash\{u, v\}$. As $G_{2}$ has a $u-v$ path, we have $d_{G}(u) \geqslant d_{G_{1}+u v}(u)$ and $d_{G}(v) \geqslant d_{G_{1}+u v}(v)$. Thus $w \in W\left(G_{1}+u v\right) \subseteq W(G)$.

By definition, $G-W(G)$ has a component $H$ such that $W(G) \subseteq N(H)$. Since $w \in$ $W(G) \cap\left(V\left(G_{1}\right) \backslash\{u, v\}\right)$, we have $V(H) \cap V\left(G_{1}\right) \neq \emptyset$. We will show that $G_{1}+u v \in \Upsilon$ in two cases.

Case 1: $V(H) \cap\{u, v\} \neq \emptyset$.

Assume that $v \in V(H)$ in this case. As $\left(W(G) \cap V\left(G_{1}\right)\right) \backslash\{u\} \subseteq N\left(V(H) \cap V\left(G_{1}\right)\right)$ and $u$ is adjacent to $v$ in $G_{1}+u v, W(G) \cap V\left(G_{1}\right)$ is dominated by $V(H) \cap V\left(G_{1}\right)$ in $G_{1}+u v$. Since $W\left(G_{1}+u v\right) \subseteq W(G) \cap V\left(G_{1}\right), W\left(G_{1}+u v\right)$ is dominated by $V(H) \cap V\left(G_{1}\right)$ in $G_{1}+u v$. Note that the subgraph of $G_{1}+u v$ induced by $V(H) \cap V\left(G_{1}\right)$ is connected no matter whether $u \in W(G)$. Thus $G_{1}+u v \in \Upsilon$, a contradiction. 
Case 2: $V(H) \cap\{u, v\}=\emptyset$.

In this case, we have $V(H) \cap V\left(G_{2}\right)=\emptyset$, as $H$ is connected and $V(H) \cap V\left(G_{1}\right) \neq$ $\emptyset$. Let $P$ be a shortest $u-v$ path in $G_{2}$. So $P$ is an induced subgraph of $G_{2}$. Let $V\left(G_{2}\right) \backslash V(P)=\left\{x_{1}, x_{2}, \ldots, x_{k}\right\}$. By Lemma 82, we have $G-\left\{x_{1}, x_{2}, \ldots, x_{k}\right\} \in \Upsilon$ and $P$ is a path in $G-\left\{x_{1}, x_{2}, \ldots, x_{k}\right\}$, contradicting the assumption on the minimality of $|E(G)|$ if $k>0$. Thus $k=0$. So $V\left(G_{2}\right)=V(P)$. By Lemma 83, repeating contracting $|E(P)|-1$ edges in $P$ yields that $G_{1}+u v \in \Upsilon$, a contradiction again.

Thus we complete the proof.

The next result from [2] will also be applied in the proof of the main result.

Lemma 10. Let $G=(V, E)$ be a non-separable graph with $|V| \geqslant 3$ and $x \in V$ with $d(x) \leqslant 3$. If $G-e$ is non-separable for every edge $e$ incident with $x$, then $G / e^{\prime}$ is also non-separable for every edge $e^{\prime}$ incident with $x$.

Now we are going to establish the main result in this section.

Theorem 11. $Q(G, \lambda)>0$ for all bridgeless graphs $G \in \Upsilon$ and for all real $\lambda \in(1,2)$.

Proof. We shall apply Theorem 6 to prove this result. Let $\beta=2, \mathcal{S}$ be the family of bridgeless graphs in $\Upsilon$ and $\mathcal{S}^{\prime}$ be the set of non-separable graphs $G=(V, E)$ with $|V| \leqslant 2$ and $E \neq \emptyset$, i.e., $\mathcal{S}^{\prime}=\{L\} \cup\left\{Z_{j}: j \geqslant 2\right\}$, where $L$ is the graph with one vertex and one loop and $Z_{j}$ is the graph with two vertices and $j$ parallel edges joining the two vertices. Note that $F(L, \lambda)=\lambda-1$ and $F\left(Z_{j}, \lambda\right)=\left((\lambda-1)^{j}+(-1)^{j}(\lambda-1)\right) / \lambda$. So it is clear that $Q(L, \lambda)=\lambda-1>0$ and

$$
Q\left(Z_{j}, \lambda\right)=(-1)^{j-2} \frac{(\lambda-1)^{j}+(-1)^{j}(\lambda-1)}{\lambda}=\frac{(\lambda-1)\left(1-(1-\lambda)^{j-1}\right)}{\lambda}>0
$$

for every $\lambda \in(1,2)$. Lemma 85 also implies that condition (ii) in Theorem 6 is satisfied. We need only to show that condition (iii) in Theorem 6 is also satisfied.

Suppose that there exists a non-separable graph $G$ in $\mathcal{S} \backslash \mathcal{S}^{\prime}$ which does not satisfy condition (iii) in Theorem 6. Thus none of conditions (a), (b), (c) of (iii) in Theorem 6 is satisfied for $G$. We will get a conclusion that such a graph $G$ does not exist and so the proof is completed by Theorem 6 .

Since $G \in \mathcal{S} \backslash \mathcal{S}^{\prime}$ and $G$ is non-separable, we have $|V(G)| \geqslant 3$. Let $W(G)=$ $\left\{x_{1}, x_{2}, \ldots, x_{k}\right\}$, where $k=|W(G)|$. By definition, $G-W(G)$ contains a component $H$ such that $W(G) \subseteq N(H)$. We now prove the following claims.

Claim 1: $G-e$ is non-separable for every $e \in E(G)$.

Suppose that $G-e$ is separable for some edge $e=u_{1} u_{2}$ of $G$. Let $v$ be any cut-vertex of $G-e$, as shown in Figure 2. As condition (a) of (iii) in Theorem 6 is not satisfied, either $G_{1}$ or $G_{2}$ does not belong to $\mathcal{S}$, where $G_{1}$ and $G_{2}$ are the graphs stated in Lemma 3. However, both $G_{1}$ and $G_{2}$ have no bridges and Lemma 9 implies that both graphs belong to $\Upsilon$, and so both belong to $\mathcal{S}$, a contradiction. Hence Claim 1 holds.

Claim 2: For every $u \in V(G) \backslash W(G)$ (i.e., $d(u) \leqslant 3$ ), $u$ is not incident with parallel edges. 
Suppose that $d(u) \leqslant 3$ and $u$ is incident with parallel edges $e_{1}$ and $e_{2}$. As $G$ is nonseparable and $|V(G)| \geqslant 3$, we have $d(u)=3$ and $u$ is incident with an edge $e$ which is not parallel to $e_{1}$ and $e_{2}$. Thus $G-e$ is separable, contradicting Claim 1.

Claim 3: For any edge $e$, if at least one end of $e$ does not belong to $W(G)$, then $G / e$ is non-separable.

Let $e$ be any edge in $G$. Assume that $u$ is one end of $e$ with $u \in V(G) \backslash W(G)$. So $d(u) \leqslant 3$. This claim then follows directly from Claim 1 and Lemma 10 .

Claim 4: $H$ is the only component of $G-W(G)$.

Suppose that $G-W(G)$ has a component $H_{1}$ different from $H$. As $G$ is connected, there exists an edge $e$ with one end $u \in V\left(H_{1}\right)$ and another end $v \in W(G)$. By Claims 1 and 3, both $G-e$ and $G / e$ are non-separable. It is also clear that both $G-e$ and $G / e$ belong to $\Upsilon$. So condition (b) of (iii) in Theorem 6 is satisfied, a contradiction.

Claim 5: For any $z \in V(H)$, if $N(z) \cap W(G) \neq \emptyset$, then either $H-z$ is disconnected or $N\left(x_{i}\right) \backslash W(G)=\{z\}$ for some $x_{i} \in W(G)$.

Suppose that this claim fails. Then $N(z) \cap W(G) \neq \emptyset, H-z$ is connected and $N(x) \backslash W(G) \neq\{z\}$ for all $x \in W(G)$.

Assume that $e$ is an edge joining $z$ and $x_{j} \in W(G)$. By Claim 2, $e$ is the only edge joining $z$ and $x_{j}$. By Claims 1 and 3 , both $G-e$ and $G / e$ are non-separable. Since $N(x) \backslash W(G) \neq\{z\}$ for all $x \in W(G)$, every vertex in $W(G)$ is dominated by $H$ in the graph $G-e$ and so $G-e \in \Upsilon$. Note that $W(G / e)=W(G)$, where $x_{j}$ is considered as the new vertex in $G / e$ when $G / e$ is produced by contracting $e$. Also note that $H-z$ is the subgraph of $G / e-W(G / e)$. Again as $N(x) \backslash W(G) \neq\{z\}$ for all $x \in W(G)$, every vertex in $W(G / e)$ is dominated by $H-z$. Since $H-z$ is connected, we have $G / e \in \Upsilon$. Now we have show that both $G-e$ and $G / e$ are non-separable and belong to $\Upsilon$, and so both belong to $\mathcal{S}$ and condition (b) of (iii) in Theorem 6 is satisfied, a contradiction.

Claim 6: $x_{i}$ and $x_{j}$ are not adjacent for all $i, j: 1 \leqslant i<j \leqslant k$.

Suppose that $x_{1}$ and $x_{2}$ are adjacent. There are two cases: $x_{1}$ and $x_{2}$ are joined by parallel edges or a single edge.

Case 1: $x_{1}$ and $x_{2}$ are joined by exactly $s$ edges, where $s \geqslant 2$.

Let $e_{1}, e_{2}, \ldots, e_{s}$ be the $s$ parallel edges joining $x_{1}$ and $x_{2}$. Let $G_{1}$ be the graph obtained from $G$ by deleting all edges $e_{1}, e_{2}, \ldots, e_{s}$ and $G_{2}$ be the graph with $V\left(G_{2}\right)=\left\{x_{1}, x_{2}\right\}$ and $E\left(G_{2}\right)=\left\{e_{1}, e_{2}, \ldots, e_{s}\right\}$. As $G$ is non-separable and $|V(G)| \geqslant 3, G_{1}$ is connected.

It is obvious that both $G_{2}$ and $G_{2}+x_{1} x_{2}$ belong to $\mathcal{S}$. By Lemma $9, G_{1}+x_{1} x_{2} \in \Upsilon$. It does not have bridges and so $G_{1}+x_{1} x_{2} \in \mathcal{S}$. By Lemma 81, $G_{1} \in \Upsilon$. If $G_{1}$ has a bridge $e$, then as $G$ is non-separable, $x_{1}$ or $x_{2}$ is a cut-vertex of $G-e$, contradicting Claim 1 . Thus $G_{1} \in \mathcal{S}$. Now both $G_{i}$ and $G_{i}+x_{1} x_{2}$ belong to $\mathcal{S}$ for $i=1,2$. Since condition (c) of (iii) in Theorem 6 is not satisfied, $b\left(G_{1}\right)+b\left(G_{2}\right)$ is odd. As $b\left(G_{2}\right)=1$, we have $b\left(G_{1}\right) \geqslant 2$. Thus $x_{1}$ and $x_{2}$ are contained in different blocks of $G_{1}$ as shown in Figure 4, where $u$ is a cut-vertex of $G_{1}$.

If $u \notin W(G)$, then $d(u)=2$ or $d(u)=3$. But, in both cases, $G_{1}$ has a bridge $e$ which is incident with $u$, contradicting $G_{1} \in \mathcal{S}$. Thus $u \in W(G)$. As $H$ is connected and $u, x_{1}, x_{2} \notin V(H), x_{s} \notin N(H)$ for some $s \in\{1,2\}$, a contradiction.

Case 2: $x_{1}$ and $x_{2}$ are joined by a single edge $e$. 


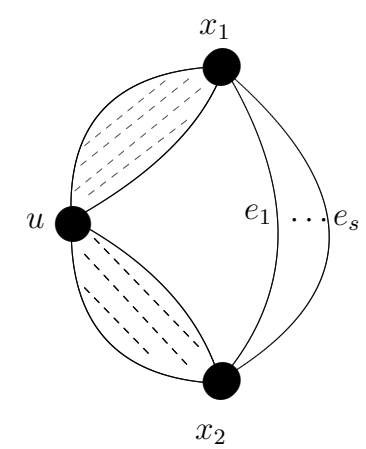

Figure 4: $u$ is a cut-vertex of $G_{1}$

Now suppose that $e=x_{1} x_{2}$ is a simple edge. By Lemma 81, both $G-e$ and $G / e$ belong to $\Upsilon$. It is clear that $G / e$ has no bridges and so it belongs to $\mathcal{S}$. By Claim 1 , $G-e$ is non-separable, and so $G-e \in \mathcal{S}$. As $G-e$ is non-separable and Condition (b) of (iii) in Theorem 6 is not satisfied, $G / e$ is separable, i.e., $\left\{x_{1}, x_{2}\right\}$ is a cut-set of $G$. Since $H$ is connected and $\left\{x_{1}, x_{2}\right\} \subseteq W(G), H$ is a subgraph of some component $H^{\prime}$ of $G-\left\{x_{1}, x_{2}\right\}$. Let $G^{\prime}$ be a component of $G-\left\{x_{1}, x_{2}\right\}$ different from $H^{\prime}$. Then $V\left(G^{\prime}\right) \nsubseteq N(H)$, contradicting the fact that $V\left(G^{\prime}\right) \subseteq W(G) \subseteq N(H)$.

Claim 7: $G$ does not exist.

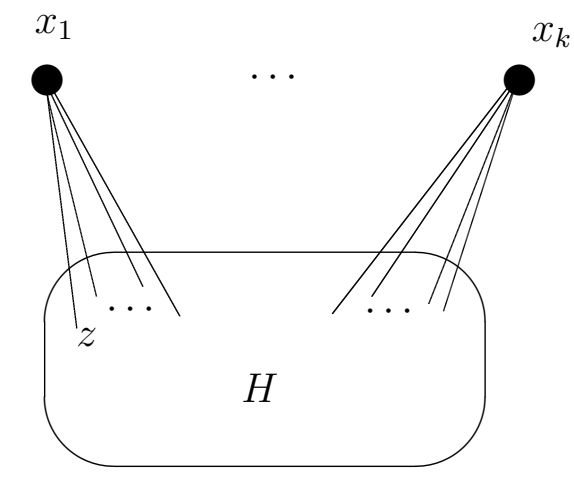

Figure 5: $\quad N\left(x_{i}\right) \subseteq V(H)$ for all $i$

By Claims 2 and $6, G$ has no parallel edges. Then Claims 4 and 6 yield that $N\left(x_{i}\right) \subseteq$ $V(H)$ and $\left|N\left(x_{i}\right) \cap V(H)\right|=d\left(x_{i}\right) \geqslant 4$ for all $i=1,2, \ldots, k$, as shown in Figure 5. Thus Claim 5 implies that each vertex $z \in N(W(G)) \subseteq V(H)$ is a cut-vertex of $H$. Thus $H$ is separable. Let $B$ be a block of $H$ which contains only one cut-vertex of $H$, say $y$. For each $z \in V(B) \backslash\{y\}$, as $z$ is not a cut-vertex of $H, z \notin N(W(G))$. Thus $B$ is a block of $G$, contradicting the fact that $G$ is non-separable. Hence Claim 7 holds.

By Claim 7, we know that every non-separable graph in $\mathcal{S} \backslash \mathcal{S}^{\prime}$ satisfies condition (iii) in Theorem 6. Thus $Q(G, \lambda)>0$ for all $G \in \mathcal{S}$ and all real $\lambda \in(1,2)$ by Theorem 6 .

We end this section by applying Theorem 11 to get a result on chromatic roots of plane graphs. Note that for any plane graph $G$, we have $P(G, \lambda)=\lambda F\left(G^{*}, \lambda\right)$. Thus 
every non-zero chromatic root of $G$ is a flow root of $G^{*}$.

Let $\mathcal{S}$ be a set of triangles in a graph $G$. A triangle-path of $\mathcal{S}$ is a sequence of distinct triangles $T_{1}, T_{2}, \ldots, T_{m}$ in $\mathcal{S}$ such that $E\left(T_{i}\right) \cap E\left(T_{i+1}\right) \neq \emptyset$ for all $1 \leqslant i \leqslant m-1$.

Corollary 12. For a connected plane graph $G$, if $G$ contains a set $\mathcal{S}$ of triangular faces such that every two members in $\mathcal{S}$ are connected by a triangle-path of $\mathcal{S}$ and every face of $G$ bounded by more than three edges is adjacent to some member of $\mathcal{S}$, then $G$ has no chromatic roots in $(1,2)$.

For example, the following plane graph has no chromatic roots in $(1,2)$.

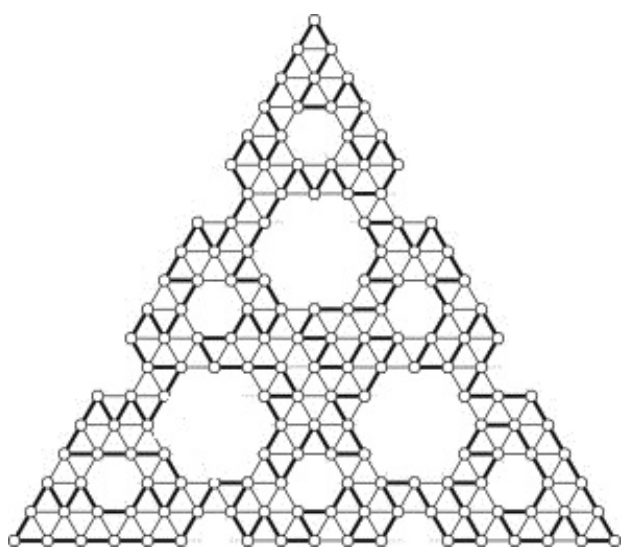

Figure 6: A plane graph without real chromatic root in $(1,2)$

\section{Construct graphs which have no flow roots in $(1,2)$}

In this section, we present two ways of constructing graphs with the property that they have no flow roots in $(1,2)$. Some graphs constructed by these two methods do not belong to $\Upsilon$.

Theorem 13. Let $G_{1}, G_{2}, \ldots, G_{k}$, where $k \geqslant 2$, be vertex-disjoint non-separable graphs which have no flow roots in $(1,2)$. Assume that $u_{i}, v_{i}$ are distinct vertices in $G_{i}$ such that $G_{i}+u_{i} v_{i}$ also has no flow roots in $(1,2)$ for all $i=1,2, \ldots, k$. If $G$ is obtained by one of the following constructions, where $k$ is even for (ii), then $G$ has no flow roots in $(1,2)$ :

(i) identifying $u_{1}, u_{2}, \ldots, u_{k}$ and identifying $v_{1}, v_{2}, \ldots, v_{k}$ respectively;

(ii) identifying $u_{i}$ and $v_{i+1}$ for all $i=1,2, \ldots, k$, where $v_{k+1}=v_{1}$.

Proof. (i) For given graphs $G_{1}, G_{2}, \ldots, G_{k}$ and any integers $s \geqslant 0$ and $0 \leqslant m \leqslant k$, let $H_{m, s}$ denote the graph obtained from $G_{1}, G_{2}, \ldots, G_{m}$ by identifying $u_{1}, u_{2}, \ldots, u_{m}$ and identifying $v_{1}, v_{2}, \ldots, v_{m}$ respectively and adding $s$ parallel edges $e_{1}, e_{2}, \ldots, e_{s}$ joining $u$ and $v$, where $u$ (resp. $v$ ) is the vertex obtained after identifying $u_{1}, u_{2}, \ldots, u_{k}$ (resp. 


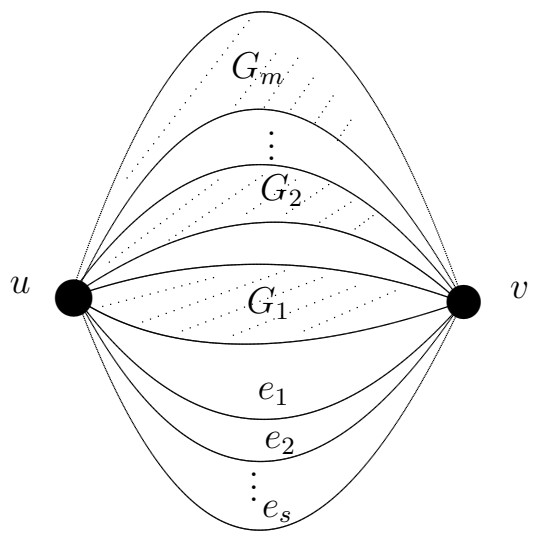

Figure 7: Graph $H_{m, s}$

$\left.v_{1}, v_{2}, \ldots, v_{k}\right)$, as shown in Figure 7 . We shall show by induction on $m$ that $Q\left(H_{m, s}, \lambda\right)>0$ for all $\lambda \in(1,2)$ whenever $m>0$ or $s \geqslant 2$.

Note that

$$
F\left(H_{0, s}, \lambda\right)=\left((\lambda-1)^{s}+(-1)^{s}(\lambda-1)\right) / \lambda .
$$

So $Q\left(H_{0,1}, \lambda\right)=0$ and when $s \geqslant 2$,

$$
Q\left(H_{0, s}, \lambda\right)=(-1)^{s-2}\left((\lambda-1)^{s}+(-1)^{s}(\lambda-1)\right) / \lambda=(\lambda-1)\left(1+(-1)^{s}(\lambda-1)^{s-1}\right) / \lambda>0 .
$$

Now assume that $m \geqslant 1$ and $s \geqslant 0$ and assume that $Q\left(H_{j, t}, \lambda\right)>0$ for all $0 \leqslant j \leqslant$ $m-1$, where $t \geqslant 2$ whenever $j=0$. If $m=1$, we may assume that $s \geqslant 2$, as $H_{1,0}$ is $G_{1}$ and $H_{1,1}$ is $G_{1}+u v$. By Lemma 4, we have

$$
F\left(H_{m, s}, \lambda\right)=F\left(G_{m}+u v, \lambda\right) F\left(H_{m-1, s}+u v, \lambda\right) /(\lambda-1)+F\left(G_{m}, \lambda\right) F\left(H_{m-1, s}, \lambda\right),
$$

i.e.,

$$
F\left(H_{m, s}, \lambda\right)=F\left(G_{m}+u v, \lambda\right) F\left(H_{m-1, s+1}, \lambda\right) /(\lambda-1)+F\left(G_{m}, \lambda\right) F\left(H_{m-1, s}, \lambda\right) .
$$

Since $G_{m}, G_{m}+u v, H_{m-1, s}$ and $H_{m-1, s+1}$ are all non-separable, we have

$$
Q\left(H_{m, s}, \lambda\right)=Q\left(G_{m}+u v, \lambda\right) Q\left(H_{m-1, s+1}, \lambda\right) /(\lambda-1)+Q\left(G_{m}, \lambda\right) Q\left(H_{m-1, s}, \lambda\right) .
$$

By the given conditions and inductive assumption, $Q\left(G_{m}, \lambda\right), Q\left(G_{m}+u v, \lambda\right), Q\left(H_{m-1, s}, \lambda\right)$ and $Q\left(H_{m-1, s+1}, \lambda\right)$ are all positive for all $\lambda \in(1,2)$. Hence $Q\left(H_{m, s}, \lambda\right)>0$ for all $\lambda \in(1,2)$. Thus the graph constructed in (i), i.e., $H_{k, 0}$ has no flow roots in $(1,2)$. 
(ii) By Lemmas 4, 2 and 3, it can be shown that

$$
F(G, \lambda)=\frac{1}{(\lambda-1)^{k-1}} \prod_{1 \leqslant i \leqslant k} F\left(G_{i}+u_{i} v_{i}, \lambda\right)+\prod_{1 \leqslant i \leqslant k} F\left(G_{i}, \lambda\right) .
$$

Note that

$$
\sum_{1 \leqslant i \leqslant k} p\left(G_{i}+u_{i} v_{i}\right)=\sum_{1 \leqslant i \leqslant k}\left(\left|E\left(G_{i}+u_{i} v_{i}\right)\right|-\left|V\left(G_{i}+u_{i} v_{i}\right)\right|\right)=(k+|E(G)|)-(k+|V(G)|)=p(G)
$$

and

$$
\sum_{1 \leqslant i \leqslant k} p\left(G_{i}\right)=\sum_{1 \leqslant i \leqslant k}\left(\left|E\left(G_{i}\right)\right|-\left|V\left(G_{i}\right)\right|\right)=|E(G)|-(k+|V(G)|)=p(G)-k .
$$

Since $k$ is even, we have

$$
Q(G, \lambda)=\frac{1}{(\lambda-1)^{k-1}} \prod_{1 \leqslant i \leqslant k} Q\left(G_{i}+u_{i} v_{i}, \lambda\right)+\prod_{1 \leqslant i \leqslant k} Q\left(G_{i}, \lambda\right) .
$$

By the given condition and Corollary $5, Q\left(G_{i}, \lambda\right), Q\left(G_{i}+u_{i} v_{i}, \lambda\right)$ and $Q\left(G / u_{i} v_{i}, \lambda\right)$ are all positive for all $\lambda \in(1,2)$. Then (16) yields that $Q(G, \lambda)>0$ for all $\lambda \in(1,2)$.

Assume that $d_{G_{i}}\left(u_{i}\right) \geqslant 2$ and $d_{G_{i}}\left(v_{i}\right) \geqslant 2$ for all $i=1,2, \ldots, k$. If $W\left(G_{i}\right) \backslash\left\{u_{i}, v_{i}\right\} \neq \emptyset$ for at least two $i$ 's, the graphs constructed by Theorem 13 (i) do not belong to $\Upsilon$. If $k \geqslant 3$, then the graphs constructed by Theorem 13 (ii) do not belong to $\Upsilon$. Hence some graphs constructed by Theorem 13 do not belong to $\Upsilon$ but have no flow roots in $(1,2)$.

Note that if $k$ is odd, the graphs obtained in Theorem 13 (ii) may have flow roots in $(1,2)$. For example, the graph shown in Figure 1 has a flow root in $(1,2)$. However, if each $G_{i}$ satisfies the extra condition that $G_{i} / u_{i} v_{i}$ has no flow root in $(1,2)$ and $b\left(G_{i} / u_{i} v_{i}\right)$ is odd, then the graphs constructed in Theorem 13 (ii) have no flow root in $(1,2)$.

Theorem 14. Let $G_{1}, G_{2}, \ldots, G_{k}$ be vertex-disjoint non-separable graphs which have no flow roots in $(1,2)$. Assume that $u_{i}, v_{i}$ are distinct vertices in $G_{i}$ such that both $G_{i} / u_{i} v_{i}$ and $G_{i}+u_{i} v_{i}$ also have no flow roots in $(1,2)$ and $b\left(G_{i} / u_{i} v_{i}\right)$ is odd for all $i=1,2, \ldots, k$. If $G$ is obtained from $G_{1}, G_{2}, \ldots, G_{k}$ by identifying $u_{i}$ and $v_{i+1}$ for all $i=1,2, \ldots, k$, where $v_{k+1}=v_{1}$, then $G$ has no flow roots in $(1,2)$.

Proof. By Theorem 13, we just need to consider the case that $k \geqslant 3$ is odd.

Since $k$ is odd, from the proof of Theorem 13 (ii), we have

$$
Q(G, \lambda)=\frac{1}{(\lambda-1)^{k-1}} \prod_{1 \leqslant i \leqslant k} Q\left(G_{i}+u_{i} v_{i}, \lambda\right)-\prod_{1 \leqslant i \leqslant k} Q\left(G_{i}, \lambda\right) .
$$

Next we show that $Q\left(G_{i}+u_{i} v_{i}, \lambda\right)-Q\left(G_{i}, \lambda\right)=Q\left(G_{i} / u_{i} v_{i}, \lambda\right)$ for each $i$. By $(2)$,

$$
F\left(G_{i}+u_{i} v_{i}, \lambda\right)+F\left(G_{i}, \lambda\right)=F\left(G_{i} / u_{i} v_{i}, \lambda\right) .
$$


Observe that

$$
\begin{aligned}
p\left(G_{i} / u_{i} v_{i}\right) & =\left|E\left(G_{i} / u_{i} v_{i}\right)\right|-\left|V\left(G_{i} / u_{i} v_{i}\right)\right|+b\left(G_{i} / u_{i} v_{i}\right)-1 \\
& =\left|E\left(G_{i}\right)\right|-\left(\left|V\left(G_{i}\right)\right|-1\right)+b\left(G_{i} / u_{i} v_{i}\right)-1 \\
& =p\left(G_{i}\right)+b\left(G_{i} / u_{i} v_{i}\right)
\end{aligned}
$$

and

$$
p\left(G_{i}+u_{i} v_{i}\right)=\left|E\left(G_{i}+u_{i} v_{i}\right)\right|-\left|V\left(G_{i}+u_{i} v_{i}\right)\right|=\left|E\left(G_{i}\right)\right|+1-\left|V\left(G_{i}\right)\right|=p\left(G_{i}\right)+1 .
$$

Since $b\left(G_{i} / u_{i} v_{i}\right)$ is odd, we have

$$
Q\left(G_{i}+u_{i} v_{i}, \lambda\right)-Q\left(G_{i}, \lambda\right)=Q\left(G_{i} / u_{i} v_{i}, \lambda\right)
$$

By the given condition and Corollary $5, Q\left(G_{i}, \lambda\right), Q\left(G_{i}+u_{i} v_{i}, \lambda\right)$ and $Q\left(G_{i} / u_{i} v_{i}, \lambda\right)$ are all positive for all $\lambda \in(1,2)$. Thus $Q\left(G_{i}+u_{i} v_{i}, \lambda\right)>Q\left(G_{i}, \lambda\right)>0$ for all $\lambda \in(1,2)$ and $i=1,2, \ldots, k$. Since $0<\lambda-1<1$ for $\lambda \in(1,2)$, by $(17)$, we have $Q(G, \lambda)>0$ for all $\lambda \in(1,2)$.

\section{Acknowledgements}

The author wishes to thank the referees for their very helpful comments and suggestions. He also wishes to thank the department of Mathematics at Xiamen University where this work was partially finished for its hospitality during the author's visit to this university in the summer of 2014.

\section{References}

[1] G.D. Birkhoff and D.C. Lewis. Chromatic polynomials. Trans. Amer. Math. Soc. 60:355-451, 1946.

[2] F. M. Dong. On zero-free intervals of flow Polynomials. J. Combin. Theory Ser. B 111: 181-200, 2015.

[3] B. Jackson. A zero-free interval for chromatic polynomials of graphs. Combin. Probab. Comput. 2: 325-336, 1993.

[4] B. Jackson. Zeros of chromatic and flow polynomials of graphs. J. Geom. 76: 95-109, 2003.

[5] B. Jackson. A zero-free interval for flow polynomials of near-cubic graphs. Combin. Probab. Comput. 16(1): 85-108, 2007.

[6] B. Jackson. A zero-free interval for flow polynomials of cubic graphs. J. Combin. Theory Ser. B 97: 127-143, 2007.

[7] J. L. Jacobsen and J. Salas. Is the five-flow conjecture almost false? J. Combin. Theory Ser. B 103: 532-565, 2013. 
[8] C.D. Wakelin. Chromatic Polynomials. Ph.D. Thesis. University of Nottingham, 1994.

[9] E.G. Whitehead Jr. and L.C. Zhao. Cutpoints and the chromatic polynomial. J. Graph Theory 8: 371-377, 1984.

[10] D.R. Woodall. Zeros of chromatic polynomials. Combinatorial Survey, Proc. Sixth British Combin. Conf. (ed. P.J. Cameron), Academic Press: 199-223, 1997.

[11] W.T. Tutte. Graph Theory. Addison-Wesley, Reading, Mass., 1984. 